\title{
Irregular narrow QRS complex tachycardia with intermittent atrioventricular dissociation: What is the mechanism?
}

\author{
Atsushi Doi ${ }^{1}$, Naoko Miyazaki ${ }^{1}$, Tomohiko Goda ${ }^{1}$, Haruya Yamane ${ }^{1}$, Kei Tanaka ${ }^{1}$, Ryo \\ Araki $^{1}$, Fumi Sato ${ }^{1}$, and Takayuki Yamada ${ }^{1}$ \\ ${ }^{1}$ Otemae Hospital
}

May 11, 2020

Irregular narrow QRS complex tachycardia with intermittent atrioventricular dissociation: What is the mechanism?

Atsushi Doi, MD, PhD; Naoko Miyazaki, MD; Tomohiko Goda, MD; Haruya Yamane, MD; Kei Tanaka, MD; Ryo Araki, MD,PhD; Fumi Sato, MD, PhD; Takayuki Yamada, MD.

Department of Cardiovascular Medicine, Otemae Hospital, 1-5-34 Otemae, Chuo-ku, Osaka, 540-0008, Japan.

Correspondence to Atsushi Doi, MD, PhD. Department of Cardiovascular Medicine, Otemae Hospital, 1-5-34 Otemae, Chuo-ku, Osaka, 540-0008, Japan.

Tel: 81-6-6941-0484, Fax: 81-6-6942-2848

E-mail; m1410001@med.osaka-cu.ac.jp

\section{DISCLOSURES}

None.

\section{EP ROUNDS}

\section{Case presentation}

A 39-year-old woman with frequent palpitations was referred for radiofrequency ablation. A baseline 12lead electrocardiogram showed normal sinus rhythm. At baseline, atrio-His (AH) and His-ventricular (HV) intervals were 80 and $45 \mathrm{~ms}$, respectively. Antegrade atrioventricular (AV) nodal Wenckebach-type ventriculoatrial (VA) block was observed at a paced cycle length (CL) of $430 \mathrm{~ms}$, and dual AV nodal physiology was observed during programmed atrial extrastimulation. The earliest retrograde atrial activation during ventricular pacing was recorded at the coronary sinus (CS) ostium. Ventriculoatrial conduction was intermittent at a paced CL of $545 \mathrm{~ms}$. Para-Hisian pacing showed AV nodal pattern. A narrow QRS supraventricular tachycardia (SVT) with HV interval of $45 \mathrm{~ms}$ dependent on a jump-up in the AH interval was induced by atrial extra stimulation (Figure 1). During SVT, intermittent AV dissociation was observed, and the atrial activation sequence at transient VA conduction was identical to that during ventricular pacing. Figure 2 shows that the H-H interval fluctuated during SVT. Based on these observations, what is the mechanism of this tachycardia?

\section{Commentary}

SVT is a narrow QRS tachycardia with intermittent AV dissociation. The differential diagnosis of SVT in this patient included AV-nodal reentrant tachycardia (AVNRT) with upper common pathway block, junctional tachycardia (JT) with junctional-atrial block, intra-Hisian reentrant tachycardia, or orthodromic 
reentrant tachycardia (ORT) using a concealed nodofascicular or nodoventricular accessory pathway with nodal atrial block.

The absence of split His bundle potentials during the procedure excludes intra-Hisian reentrant tachycardia. SVT was necessarily initiated depending on a critical AH interval prolongation (Figure 1). Moreover, atrial overdrive pacing during SVT accelerated the His bundle potentials to the paced CL, and produces an atrialHis-atrial (AHA) response upon pacing cessation. These findings rule out the diagnosis of JT. ${ }^{1}$

When the H-H interval is relatively stable, no premature ventricular extrastimuli delivered during His bundle refractoriness preexcited the next His bundle potential. After infusion of isoproterenol, SVT with the same earliest atrial activation at the CS ostium with a fixed 1:1 AV relationship was induced by atrial extra stimulation with a finding of an AH jump up. A ventricular extrastimuli delivered during SVT when the His bundle was refractory did not reset the atrial cycle. A ventricular-atrial-ventricular (VAV) response was observed upon resumption of the tachycardia, on cessation of ventricular entrainment pacing, and the difference between the postpacing interval and tachycardia CL was $139 \mathrm{~ms}$, which is longer than $125 \mathrm{ms.}^{2}$ Figure 3 shows RV overdrive pacing during SVT at a faster pacing rate. During RV pacing, 2 consecutive His bundle potentials occur at the pacing CL shortly after the fourth and fifth pacing stimuli, but there were no change of the A-A intervals after those pacing stimuli, and VA block was observed after the sixth pacing stimulus. That is, RV overdrive pacing during SVT retrogradely captured the His bundle but failed to accelerate the atrium. ${ }^{3}$ These findings consistent with AVNRT, and, thus, ruled out the possibility of ORT.

We performed electroanatomical mapping to identify the earliest site of atrial activation during SVT, and observed that the arrhythmia was not inducible with disappearance of VA conduction after radiofrequency ablation at the CS ostium, consistent with successful ablation of the slow pathway. Thus, we diagnosed SVT as atypical AVNRT using the slow pathway as an anterograde limb and the slow pathway as a retrograde limb (slow-slow AVNRT) with upper common pathway block.

In this case, the fluctuation of the H-H intervals during slow-slow AVNRT with AV dissociation can be explained by the timing of the dissociated sinus rhythm; late (Figure 2A) and early (Figure 2B) coupled sinus complexes (see the asterisk in the figure). In Figure $2 \mathrm{~A}$, the second and third sinus complexes advanced the next His bundle potentials. That is, the sinus complexes delivered during the refractoriness of the His bundle anterogradely conducted down the slow pathway, reached the His bundle, conduced to the ventricle, and returned through the slow pathway. Figure 2B shows that the early atrial contraction from the sinus node advanced the immediate His bundle potential by $47 \mathrm{~ms}$ with continuation of the tachycardia. The first to third $\mathrm{H}-\mathrm{H}$ intervals were $625 \mathrm{~ms}$, and the fourth was shortened to $578 \mathrm{~ms}$ after an atrial contraction from the sinus node. The fifth $\mathrm{H}-\mathrm{H}$ interval was prolonged to $672 \mathrm{~ms}$, while the sixth and seventh $\mathrm{H}-\mathrm{H}$ intervals returned to $625 \mathrm{~ms}$. The interval from the fourth to the sixth His bundle potentials was exactly identical to twice the CL of tachycardia. Atrial excitement from the sinus node descends the fast pathway, reaches the His bundle, and conducts to the ventricle, though it does not affect the tachycardia circuit. That is, because the fast pathway was not included in the tachycardia circuit, slow-slow AVNRT continued even when the immediate His potential was advanced. We think that these might be the reason why the $\mathrm{H}-\mathrm{H}$ intervals fluctuated during slow-slow AVNRT.

\section{References}

1. Fan R, Tardos JG, Almasry I, Barbera S, Rashba EJ, Iwai S. Novel use of atrial overdrive pacing to rapidly differentiate junctional tachycardia from atrioventricular nodal reentrant tachycardia. Heart Rhythm 2011;8:840-844.

2. Ho RT, Frisch DR, Pavri BB, Levi SA, Greenspon AJ. Electrophysiological features differentiating the atypical atrioventricular node-dependent long RP supraventricular tachycardias. Circ Arrhythm Electrophysiol 2013;6:597-605.

3. Ho RT, Pietrasik G, Greenspon AJ. A narrow complex tachycardia with intermittent atrioventricular dissociation: what is the mechanism? Heart Rhythm 2014;11:2116-2119.

\section{FIGURE LEGENDS}


Figure 1A narrow QRS tachycardia dependent on a jump-up in the AH interval was induced by atrial extra stimulation. The numbers indicate the cycle lengths in ms. II, V2, V5 = surface electrogram; His d and His $\mathrm{p}=$ distal and proximal His bundle region; CS d to CSOS = distal to proximal coronary sinus ostium; $\mathrm{HRA}=$ high right atrium; $\mathrm{RV}=$ right ventricle; $\mathrm{A}$ and $\mathrm{H}=$ atrial and His bundle electrogram.Figure 2During the tachycardia, intermittent AV dissociation was observed, and the $\mathrm{H}-\mathrm{H}$ intervals fluctuated. (A) late coupled sinus complex (B) early coupled sinus complex asterisk = atrial contraction from the sinus node. The other abbreviations are as in Figure 1.Figure 3The response to the ventricular overdrive pacing during the tachycardia. arrow $=$ The pacing stimulus at CSOS did not capture the atrium. $\mathrm{rH}=$ retrograde His bundle potential. The other abbreviations are as in Figure 1.

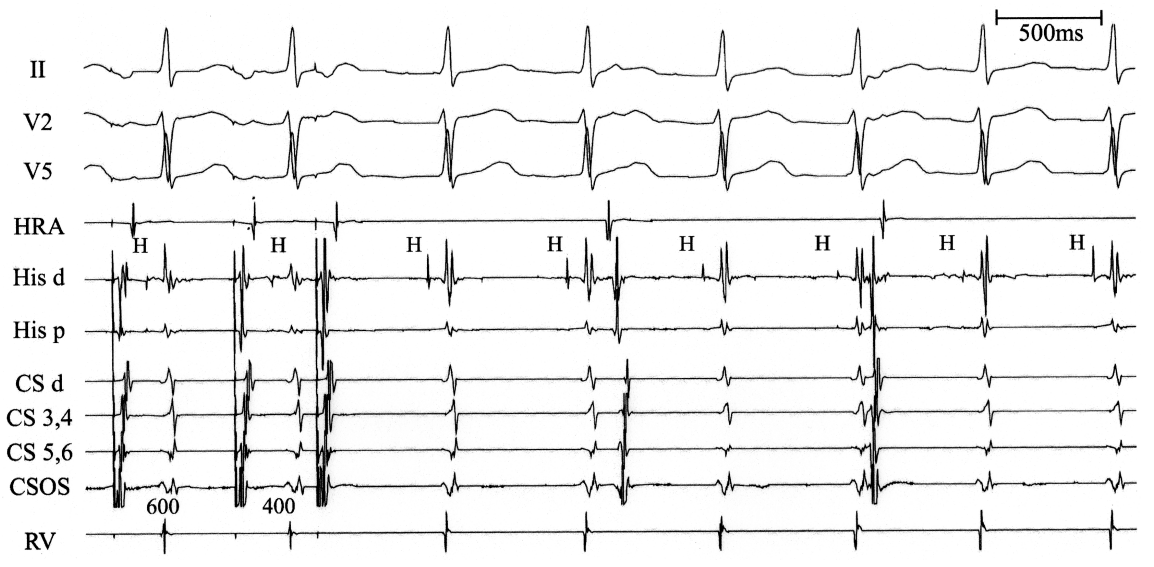


II
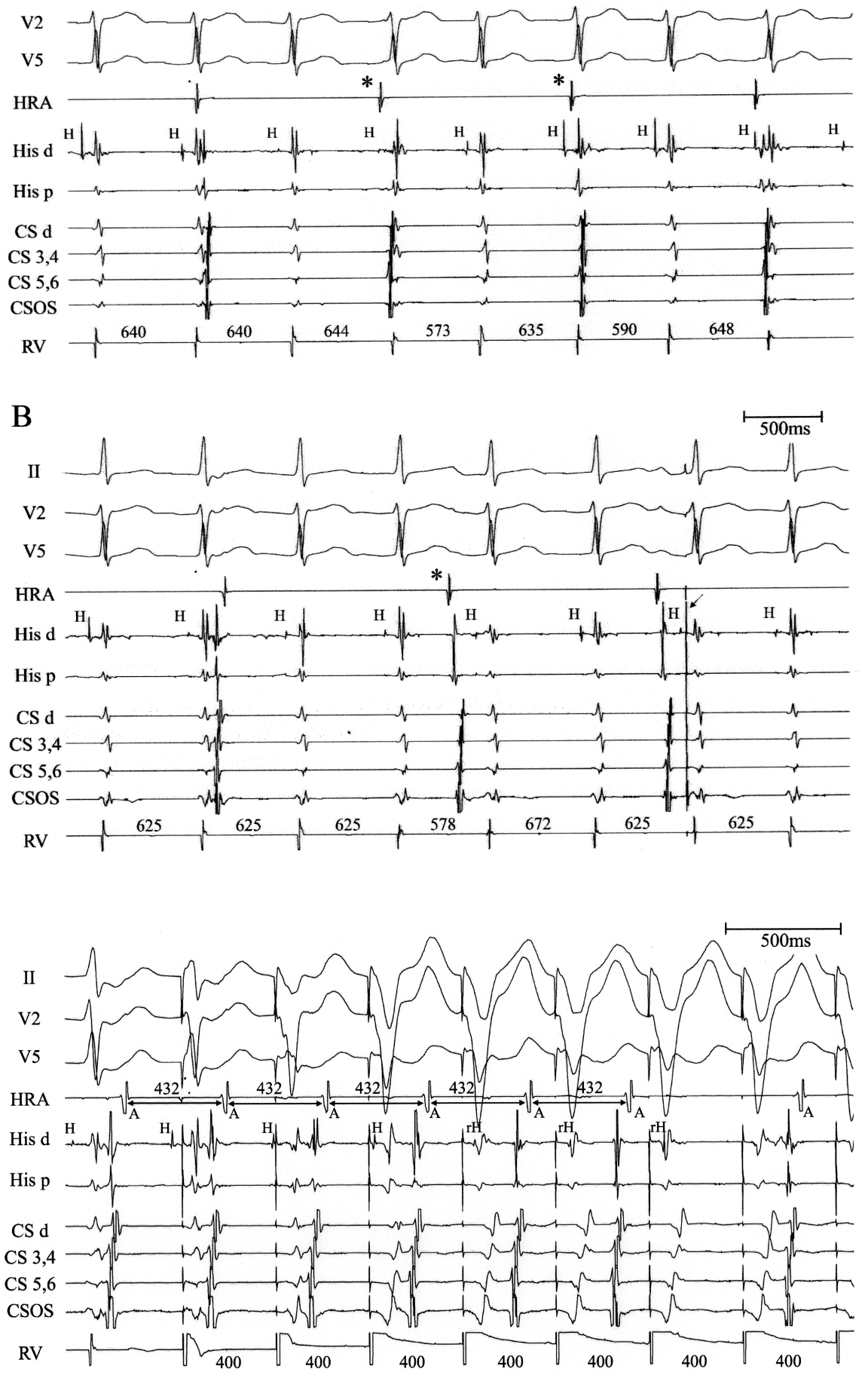